\title{
Two types of induced familiarity in the matching of letter strings
}

\author{
GARY R. KIDD, ALEXANDER POLLATSEK, and ARNOLD D. WELL \\ University of Massachusetts, Amherst, Massachusetts 01003
}

\begin{abstract}
Subjects received training with four-letter consonant strings in two different tasks over a 6-day training period. One was a paired associate learning task in which the letter strings were used as response elements, and the other was a simultaneous matching task in which subjects were required to judge whether or not pairs of the consonant strings were physically identical. In a test session in which subjects performed the matching task, stimuli from both paired associate and matching training tasks were matched more quickly than control strings that had not been seen previously. Further analyses suggested that the familiarity effect for paired associate stimuli resulted from more "holistic" processing than the familiarity effect for stimuli previously matched in training.
\end{abstract}

Since Cattell (1886), the word superiority effect has been demonstrated in many different experimental situations in attempts at its elucidation (e.g., Eichelman, 1970; Krueger, 1970; Reicher, 1969). In this paper we will concern ourselves primarily with the simultaneous matching task. The task requires subjects to decide whether or not two letter strings are physically identical. Recently, Pollatsek, Well, and Schindler (1975) found that words were compared faster than nonwords in a simultaneous matching task even for "different" responses when the difference was only one of case (e.g., SITE vs. SItE). This demonstrates that the advantage of words over nonwords in this task is due, at least in part, to visual mechanisms and not simply to verbal mechanisms (i.e., comparing word or letter names) or semantic mechanisms (i.e., comparing meanings).

One strategy for studying why the visual processing of words is faster than for nonwords is the experimental manipulation of subjects' experience with novel letter strings. This strategy offers, among other things, the opportunity for determining whether familiarity per se can mediate part of the word superiority effect. However, there has been relatively little research with induced visual familiarity effects. Baron (1974) found that accuracy in a forced-choice tachistoscopic recognition task was greater for consonant digrams that had been viewed during at least four training sessions than for consonant digrams consisting of the same letters in reverse order that were seen in only one session. Unfortunately, it is hard to directly relate this result to the familiarity effect for words since the letter strings

This research was supported in part by a grant from the University of Massachusetts honors program and alumni association. The first author is now a graduate student at Ohio State University. Reprint requests should be sent to Alexander Pollatsek, Department of Psychology, University of Massachusetts, Amherst, Massachusetts 01003. (digrams) used were shorter than most words and subjects' visual experience with the stimuli was brief.

Schindler, Well, and Pollatsek (1976) manipulated experience with six-letter consonant strings in a simultaneous matching task which extended over 16 training sessions in an attempt to induce the familiarity effect. Some letter strings were presented in each of the training sessions, while other letter strings were presented only during a single session. An advantage in matching time for those strings presented during each session was found which gradually increased during training and persisted for at least 7 weeks after training. While the persistence of the effect suggests that the mechanism involved here may be the same one responsible for the familiarity effect for common words, the kind of matching training was very different from our normal experience with words. Since the learning that took place in that experiment may have been task specific, it is important to determine whether the familiarity effect can be induced in other contexts.

The present experiment focuses on whether familiarity effects can be induced in the simultaneous matching task by training in a different kind of task that is more similar to our natural experience with words. For this purpose a paired associate task was used. Subjects learned a list of 16 paired associates following the standard study-test procedure over a 6-day training period. The possible effects of familiarity were examined in a testing session on Day 7 of the experiment by comparing matching times for response elements from the paired associate task with those for other four-letter consonant strings that subjects had never seen before. In addition, during each of the training sessions, subjects performed simultaneous matching with a third set of stimuli so that any familiarity effect for stimuli from paired associate training could be compared with that from matching training.

In order to determine whether or not meaningful associations are a relevant factor in the development of a 
familiarity effect, meaningfulness was manipulated in the paired associate training. Half of the stimulus elements used were common four-letter words and half were meaningless consonant digrams. If, meaningful associations contribute to the familiarity effect, then the effect should be larger for stimuli that were associated with common words than for those associated with meaningless digrams.

\section{METHOD}

\section{Subjects}

The subjects were 13 University of Massachusetts students. Five of the females and the five males were psychology majors who received course credits for participation. The remaining three were female volunteers, two nonpsychology undergraduates and one psychology graduate student.

\section{Apparatus and Manner of Stimulus Presentation}

A Hewlett-Packard 2114B computer was used to record subjects' responses, measure reaction times, and display the stimuli. The stimuli (capital letters) were displayed on an HP 1300A X-Y display oscilloscope. Subjects were run individually and sat approximately $1.5 \mathrm{~m}$ away from the oscilloscope screen in a dimly lit room.

In the simultaneous matching task, a display consisted of two four-letter strings, with one string directly above the other. The pair of four-letter strings subtended about $1.5 \mathrm{deg}$ of visual angle both horizontally and vertically. In the paired associate task, a display on a trial consisted of two four-letter strings in a row separated by a dash with one letter space on either side of it. The dash appeared near the center of the screen. Each letter string was of the same size as a letter string in the matching task. For the test phase, only the left-hand string was displayed, followed by the dash which was still centered.

\section{Procedure}

The experiment was run on 7 consecutive weekdays. Subjects started on different days of the week, the only restriction being that the final testing session immediately followed the 6th day of training.

Two different tasks were performed by the subjects on each of the 6 training days. The first task on each day was a simultaneous matching task, in which subjects were required to indicate whether or not two letter strings were identical by depressing one of two response keys with either the left or right index finger. Subjects were told to respond as quickly and as accurately as possible. The assignment of "same" or "different" to the response keys was counterbalanced across subjects. On each trial a "+" appeared in the center of the screen 1 sec before the strings; it served as both a warning and as a fixation point. Subjects were given feedback as to their speed and accuracy after each trial block.

The second task was a paired associate learning task. Common four-letter words were used as stimulus elements for half of the 16 paired associates and meaningless consonant digrams were used as stimulus elements for the remaining eight paired associates. All the response elements were meaningless four-letter consonant strings. The paired associates were divided into two groups based on the meaningful/meaningless distinction. Training of the meaningful and meaningless groups was alternated during the paired associate training session, with the meaningful group appearing first. Each group was seen an equal number of times.

The training in the paired associate task followed the standard study-test procedure, in which each of the eight pairs from a group was displayed for $4 \mathrm{sec}$ of study time followed by $4 \mathrm{sec}$ of off time. After all of the pairs in the group were displayed, subjects were tested for recall of each response in turn upon presentation of the appropriate stimulus element.
Table 1

The Three Orderings of the Conjugate Pairs

\begin{tabular}{lllll}
\hline & \multicolumn{4}{c}{ Serial Position } \\
\cline { 2 - 5 } & 1 & 2 & 3 & 4 \\
\hline \multirow{2}{*}{ Order 1 } & C & D & F & K \\
& J & P & H & M \\
Order 2 & K & F & D & C \\
& M & H & P & J \\
Order 3 & D & K & C & F \\
& P & M & J & H \\
\hline
\end{tabular}

In an attempt to equate the amount of training in the paired associate and matching tasks, equal numbers of presentations were given in each task. Only presentations in the study portion of the paired associate task were counted since the "response" string was not presented during the test portion. ${ }^{1}$ However, due to an oversight on the part of the experimenter, practice was not equated in this manner for the first seven subjects. These subjects saw four trial blocks of the matching task and went through the study-test procedures for the 16 paired associates only four times, for a total of 192 matching presentations and only 64 paired associate presentations per day. This was corrected for the last six subjects, who saw two trial blocks of matching and went through the paired associate list six times, for a total of 96 presentations per day in each task.

On the 7 th, or test, day of the experiment, subjects received nine 48-trial simultaneous matching blocks: three blocks using stimuli previously used in matching training, three blocks using as stimuli the response elements used in paired associate training, and three blocks using a set of control stimuli that they had not seen before. A given trial block contained stimulus pairs from only one of these three sets, and the order of the three categories of trial blocks was counterbalanced across subjects.

\section{Stimuli and Design}

The consonants comprising the letter strings used in this study were chosen from the set of four conjugate pairs shown in Table 1. Each letter string was a sequence of four consonants, one chosen from each conjugate pair. Each of the three assignments of conjugate pairs to letter positions (see Table 1) generated a set of 16 possible letter strings. Each set was assigned to one of the three experimental conditions, and this assignment was counterbalanced across subjects. That is, one set of stimuli was used as the response elements in the paired associate task, another set was used in matching training, and the remaining (control) set was only seen in the matching task on the final day.

For the matching task, "different" displays were formed (1) by randomly choosing one of the 16 strings in the appropriate stimulus set for display in the upper position and (2) by replacing one of the letters in the string with its conjugate before displaying it in the lower position.

\section{RESULTS}

The mean reaction times for the test session were calculated for each of the three stimulus sets: previously matched stimuli, paired associate stimuli, and control stimuli. It was found that both sets of stimuli dealt with during training were matched more quickly than controls. When "same" and "different" data were combined, the familiarity effect was $66 \mathrm{msec}[\mathrm{t}(12)=4.409$, $\mathrm{p}<.002, \mathrm{SE}=15.02]$ for previously matched stimuli and $54 \mathrm{msec}[\mathrm{t}(12)=2.349, \mathrm{p}<.05, \mathrm{SE}=23.08]$ for paired associate stimuli. When "same" and "different" 
data were analyzed separately, the story was much the same; only the 38 -msec familiarity effect for "different" data failed to reach significance for paired associate stimuli $[\mathrm{t}(12)=1.525, \mathrm{SE}=25.22]$.

Some of the variability in the data was due to Subjects 8-13 having had more experience with the paired associate stimuli during training than Subjects 1-7. Subjects 8-13 did in fact have a significantly larger familiarity effect for paired associate stimuli than did Subjects $1.7[\mathrm{t}(11)=2.776, \mathrm{p}<.05, \mathrm{SE}=38.88]$, and had, by themselves, a marginally significant $92-\mathrm{msec}$ familiarity effect for "different" responses $[\mathrm{t}(5)=2.441$, $\mathrm{p}<.05, \mathrm{SE}=37.63$, one-tailed $]$.

There was some evidence that paired associate stimuli may have been processed qualitatively differently than previously matched stimuli. It can be seen in Figure 1 that response times for paired associate stimuli were much less affected by type of response ("same" or "different") than were data from previously matched stimuli $[\mathrm{t}(12)=5.416, \mathrm{p}<.002, \mathrm{SE}=13.21]$. This effect was present separately for Subjects $1-7[\mathrm{t}(6)=$ 5.023, $\mathrm{p}<.01, \mathrm{SE}=17.31]$ and Subjects 8-13 $[\mathrm{t}(5)=$ $2.739, \mathrm{p}<.05, \mathrm{SE}=19.18]$.

In addition, as can be seen in Figure 2, the serial position curve for paired associate stimuli was flatter than those for previously matched or control stimuli. The slope for paired associate stimuli was $27.4 \mathrm{msec} /$ serial position, whereas for previously matched stimuli it was $56.9 \mathrm{msec} / \mathrm{serial}$ position. However, this difference just missed significance at the .05 level $[\mathrm{t}(12)=2.162$, $\mathrm{p}<.1]$.

It is perhaps surprising that the slopes of serial position curves for previously matched and control stimuli

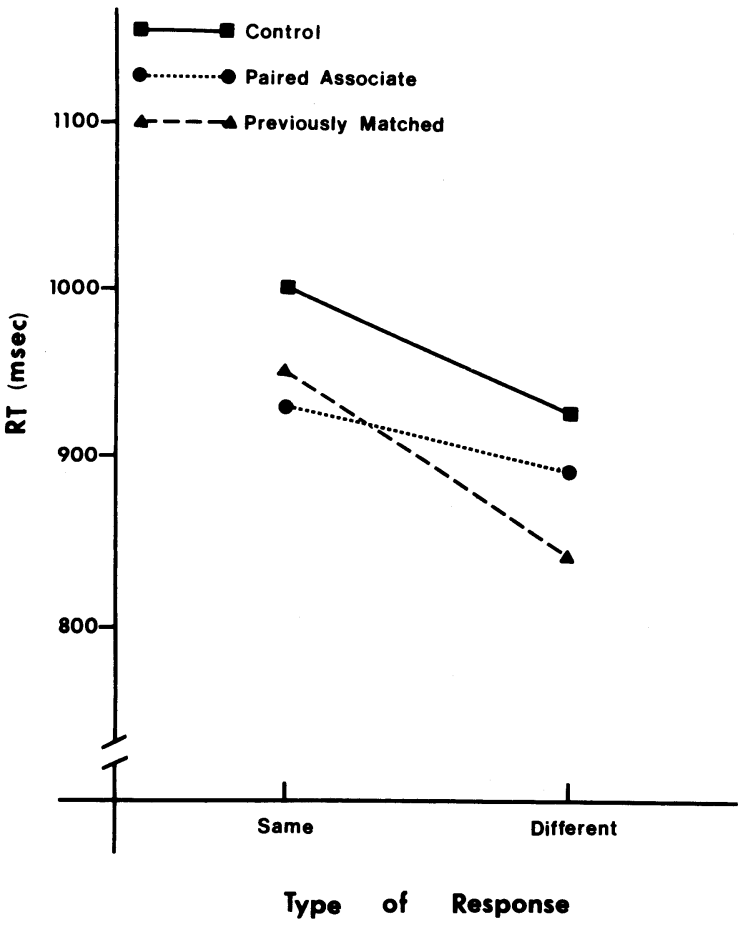

Figure 1. Reaction time as a function of "same" and "different" responses for the three experimental conditions.

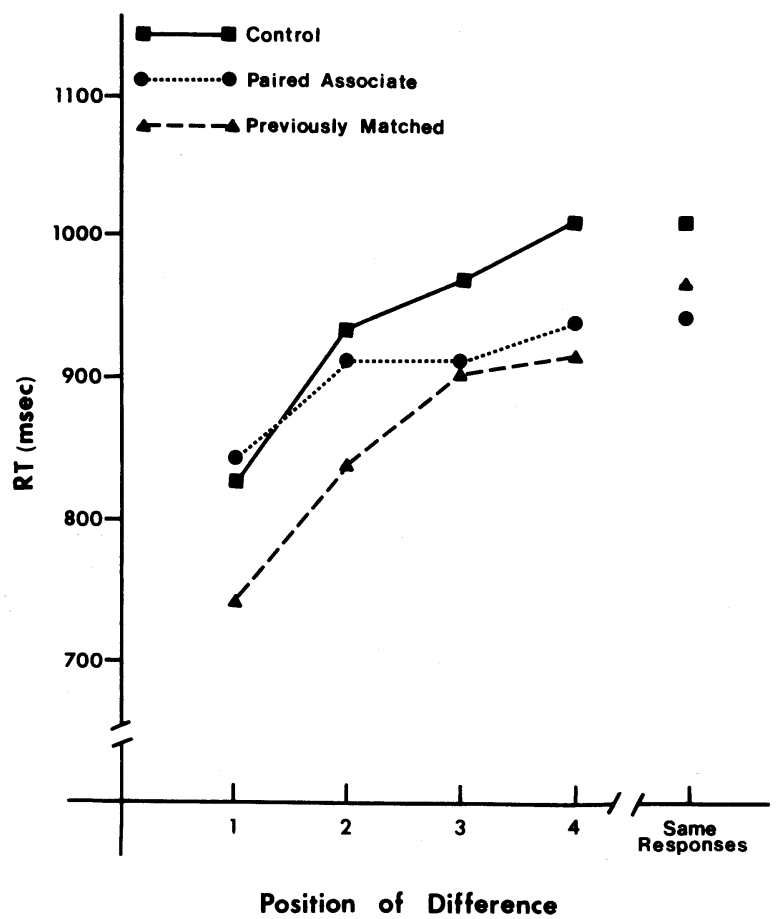

Figure 2. Serial position curves for the three experimental conditions.

did not differ. There may have been, however, a real difference in slope that was obscured by the pattern of speed-accuracy tradeoffs over the four serial positions (see error data below).

The manipulation of type of association (i.e., meaningful vs. meaningless) for the letter strings viewed in the paired associate task yielded only a very small (approximately $11.9 \mathrm{msec}$ ), insignificant $(\mathrm{t}<1)$ effect in the expected direction. There was also very little difference in the number of trials subjects took to learn meaningful vs. meaningless associations. The mean number of trials to criterion (where criterion was four consecutive correct responses) was 9.48 for meaningful pairs and 9.81 for meaningless pairs.

The number of trials to criterion seemed to be an important variable affecting both absolute reaction time and size of the familiarity effect. Reaction times were plotted as a function of trials to criterion for each subject and the slope of the best fitting line was determined. Over all 13 subjects the slope was found to be significantly greater than zero [mean slope $=6.95 \mathrm{msec} /$ trial, $t(12)=2.904, p<.05, S E=2.39$ ]. Also, a significant correlation across subjects of mean number of trials to criterion with size of the familiarity effect was found $[\mathrm{r}=.5549, \mathrm{t}(11)=2.212, \mathrm{p}<.05]$.

\section{Error Rates}

The mean error rates for the three experimental conditions were calculated separately for "same" and "different" responses and for the four positions of difference (see Table 2). (The statistical significance of difference in error was assessed using a square root transformation on the frequencies.) 
Table 2

Error Rates (Persent)

\begin{tabular}{|c|c|c|c|c|c|c|}
\hline \multirow[b]{2}{*}{ Group } & \multicolumn{2}{|c|}{ Responses } & \multicolumn{4}{|c|}{ Serial Position } \\
\hline & "Same" & $\begin{array}{c}\text { "Differ- } \\
\text { ent" }\end{array}$ & 1 & 2 & 3 & 4 \\
\hline Control & 1.82 & 4.49 & 4.70 & 4.70 & 2.99 & 5.56 \\
\hline Paired Associate & 1.71 & 4.70 & 6.41 & 5.13 & 3.85 & 3.42 \\
\hline Previously Matched & 1.39 & 1.50 & .85 & 3.85 & 1.28 & 0.00 \\
\hline
\end{tabular}

Familiarity effects observed for the previously matched and paired associate stimuli cannot be accounted for by speed-accuracy tradeoffs, as the error rate for the control condition was significantly higher than the error rate for the previously matched condition $[\mathrm{t}(12)=2.687, \mathrm{p}<.025, \mathrm{SE}=.737]$, and was virtually identical to the error rate for the paired asscciate condition. In addition, the significantly smaller difference between "same" and "different" reaction times for paired associate stimuli than that for previously matched stimuli cannot be accounted for by a speed-accuracy tradeoff, since the interaction in the error data is in the opposite direction from that predicted by such a tradeoff $[t(12)=1.755, p>.10, S E=.798]$. Furthermore, the observed difference in slopes as a function of serial position between paired associate and previously matched stimuli cannot be accounted for by a speedaccuracy tradeoff, since differences in error rates between the two conditions had no clear pattern and the Condition by Position interaction for these two conditions was insignificant $(\mathrm{F}<1)$.

There is, however, some suggestion that the lack of difference in slope between the previously matched and control stimuli may be explained by differences in error rates. There is a consistent decrease in the error rate as a function of serial position for the previously matched stimuli, while the error rate appears to rise a bit for the control condition. However, this interaction is not significant $[\mathrm{F}(3,36)=1.30, \mathrm{p}<.20, \mathrm{MSe}=.347]$.

The decrease in error rate with serial position of difference for the previously matched and paired associate stimuli is a puzzling feature of the data. The typical and more intuitive result (e.g., Well, Pollatsek, \& Schindler, 1975) is that error rate increases with serial position. The reason for the decreases in the present experiment are not clear.

\section{DISCUSSION}

The significant familiarity effect found for letter strings previously matched during training provides further support for the suggestion of Schindler et al. (1976) that repeated exposures of letter strings in a matching task result in perceptual facilitation due to the storage of specific information about the letter strings. The finding of a significant familiarity effect for letter strings previously seen only in a paired associate task extends the results of Baron (1974) and Schindler et al. (1976), who found facilitation in test situations identical to their training situations.
Another interesting finding of this study is that the processing of letter strings in the testing session seemed to be qualitatively different for stimuli seen in the paired associate task compared to stimuli seen in the matching task during training. This notion is supported by the fact that matching times for paired associate stimuli were much less affected by type of response than those for previously matched stimuli, and by the flatter "different"-response serial position curve for paired associate stimuli than for previously matched stimuli.

Of particular interest in the present experiment is the fact that the paired associate training task was a nonspeeded task that is more similar to our natural experience with real words than is matching (i.e., we examine words carefully and learn their association with some meaning). In fact, characteristics of the paired associate data (e.g., the flatter serial position curve for "different" responses) resemble the data for common words from other familiarity effect studies (e.g., Well et al., 1975).

These results suggest that paired associate stimuli may have been processed in a more "holistic" fashion than previously matched stimuli. Such data are consistent with models of the simultaneous matching task, such as that of Well et al. (1975), if one assumes that the experience of processing a letter string in a paired associate context causes it to be subsequently processed in units larger than those of strings experienced only in a matching task. If one conceives of the matching process as a serial self-terminating search for a difference which, if unsuccessful, results in a "same" response, then the closer in size the unit of processing is to the whole letter string (1) the smaller will be the difference between "same" and "different" responses and (2) the smaller will be serial position effects.

\section{REFERENCES}

BARON, J. Facilitation of perception by spelling constraints. Canadian Journal of Psychology, 1974, 28, 37-50.

Cattell, J. M. The time taken up by cerebral operations. Mind, 1886, 11, 277-292, 524-538.

Eichelman, W. H. Familiarity effects in the simultaneous matching task. Journal of Experimental Psychology, 1970, 86, $275-282$.

KRUEGER, L. E. Effect of frequency of display on speed of visual search. Journal of Experimental Psychology, 1970, 84, 495-498.

Pollatsek, A., Well, A. D., \& Schindler, R. M. Familiarity affects visual processing of words. Journal of Experimental Psychology, 1975, 1, 328-333.

Reicher, G. M. Perceptual recognition as a function of meaningfulness of stimulus material. Journal of Experimental Psychology, 1969, 81, 275-280.

Schindler, R. M., Well, A. D., \& Pollatsek, A. Inducing the familiarity effect. Perception \& Psychophysics, 1976, 19, 425-432.

Weli, A. D., Pollatsek, A., \& Schindler, R. M. Facilitation of both "same" and "different" judgments of letter strings by familiarity of letter sequence. Perception \& Psychophysics, 1975, 17, 511-520.

\section{NOTE}

1. It is not clear how to properly equate the amount of practice in the paired associate and simultaneous matching tasks since we do not really know what kind of learning is going on when subjects perform these tasks. Given this state of affairs, equating the number of trials in each task seemed to be a reasonable strategy. 